\title{
Synthesis of triacetonamine $N$-alkyl derivatives reinvestigated
}

\author{
Klaus Banert,* Katharina Fink, Manfred Hagedorn, and Frank Richter \\ Chemnitz University of Technology, Organic Chemistry, \\ Strasse der Nationen 62, 09111 Chemnitz, Germany \\ E-mail: klaus.banert@chemie.tu-chemnitz.de \\ Dedicated to Professor Rainer Beckert on his $60^{\text {th }}$ birthday
}

\begin{abstract}
The $N$-alkylated 2,2,6,6-tetramethylpiperidin-4-ones 3c-f were prepared from the acetal 6a of triacetonamine (3a) by alkylation followed by hydrolysis of the acetal functionality or alternatively from the corresponding secondary alcohol 2,2,6,6-tetramethylpiperidin-4-ol (7a) by $\mathrm{N}$-alkylation and subsequent oxidation to introduce the ketone unit. Direct alkylation of 3a was only possible by using highly reactive halides such as allyl or benzyl bromide with low yields. Treatment of phorone (5) with primary amines $\mathbf{2 c - f}$ with an alkyl group greater than methyl did not lead to the desired heterocycles 3c-f since open-chain addition products $\mathbf{8}$ and $\mathbf{9}$ were formed instead. Consequently, the reactions of acetone (1) with benzyl- or $n$-butylamine (2e,f) in the presence of calcium chloride did not generate the corresponding $N$-alkylated derivatives of $\mathbf{3 a}$.
\end{abstract}

Keywords: $N$-Alkylation, 2-aminothiazoles, 2,2,6,6-tetramethylpiperidin-4-ones, ring closure, steric hindrance, tertiary amines

\section{Introduction}

Sterically hindered amines are important compounds because of a variety of applications. Such compounds and their metal salts are particularly useful as bases in synthesis. ${ }^{1}$ Furthermore, hindered amines play an important role as precursors to persistent nitroxyl radicals, which were used for spin labeling methods. ${ }^{2}$ Such amines have recently come into industrial use in a variety of gas-treating processes. ${ }^{3}$

Numerous 2,2,6,6-tetramethylpiperidines of type 4 and free radicals derived from these heterocycles are polymerization inhibitors and thermo- and photostabilizers, known as hindered amine light stabilizers and abbreviated as HALS (Scheme 1). ${ }^{4}$ Triacetonamine (3a) is the unique starting compound for the synthesis of the desired products $4 .^{5}$ The piperidine derivative $\mathbf{3 a}$ can be prepared from acetone (1) and ammonia (2a) in the presence of an acidic catalyst like calcium 
chloride $^{6}$ or from phorone ${ }^{7}(\mathbf{5})$ and $\mathbf{2 a}$ or by other methods. ${ }^{8}$ It was claimed that treatment of $\mathbf{1}$ with $\mathbf{2 b}, \mathbf{2 e}$, or $\mathbf{2 f}$ led analogously to $\mathbf{3 b}, \mathbf{e , f}$ in 30,85 , and $15 \%$ yield, respectively. ${ }^{9}$ On the other hand, it was reported that the heterocycles $\mathbf{3 b}$-e can be prepared from $\mathbf{5}$ and primary amines $\mathbf{2 b}-\mathbf{e}^{7 \mathrm{a}, \mathrm{b}}$ Whereas synthesis of $\mathbf{3 b}$ from $\mathbf{5}$ and $\mathbf{2 b}$ was repeated by other authors several times, ${ }^{10}$ the isolation of $\mathbf{3 e}$ after the generation from $\mathbf{5}$ and $\mathbf{2 e}$ was described recently to be problematic. ${ }^{11}$ The reports on the $N$-alkylation of 3a are also contradictory. Methylation of 3a with the help of methyl iodide was successfully performed repeatedly, ${ }^{12}$ while attempted methylation of $\mathbf{3 a}$ with formaldehyde and formic acid failed. ${ }^{12 a}$ Introduction of more bulky substituents meets with increasing steric resistance. Thus, treatment of $\mathbf{3 a}$ with ethyl iodide was reported to give complicated mixtures of products instead of desired 3c. ${ }^{13}$ However, other authors claimed that the reaction of $\mathbf{3 a}$ with ethyl iodide or allyl bromide led to the corresponding hydrohalides of $\mathbf{3 c}$ and 3d, respectively. ${ }^{14}$ On the other hand, $N$-alkylation of 2,2,6,6-tetramethylpiperidines 4, which do not possess an oxo group in 4-position, for example, 4a, is more easily achieved. ${ }^{15}$

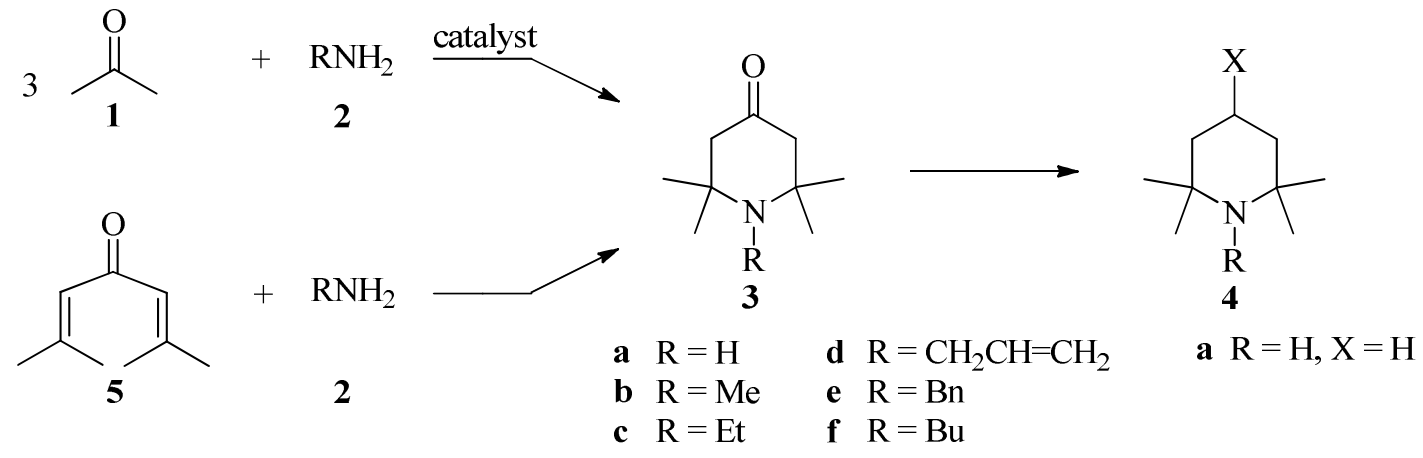

Scheme 1. Syntheses of 2,2,6,6-tetramethylpiperidines.

The aim of our work was to prepare the heterocycles $\mathbf{3 c}-\mathbf{f}$ by novel methods and to prove unequivocally their structures by spectroscopic data since these compounds were previously characterized only by elemental analyses and melting points of derivatives in most cases. Moreover, we wanted to clarify whether $\mathbf{3 c}-\mathbf{f}$ can be synthesized from $\mathbf{1}$ and $\mathbf{2}$ in the presence of acidic catalysts or by treatment of $\mathbf{5}$ with the corresponding primary amines $\mathbf{2}$.

\section{Results and Discussion}

We prepared the $N$-alkylated product $\mathbf{6 c}$ by treating the acetal $\mathbf{6 a}$, which was easily available from ketone $\mathbf{3 a},{ }^{16}$ with ethyl iodide in toluene in the presence of calcium carbonate (Scheme 2). Subsequent hydrolysis of $\mathbf{6 c}$ led to the triacetonamine derivative 3c. We tried also another route to get the product $\mathbf{3} \mathbf{c}$ because alkylation of $\mathbf{6 a}$ was only possible at high temperature and with low yield. The alcohol $\mathbf{7 a}$, which is conveniently accessible from $\mathbf{3 a},{ }^{12 \mathrm{a}}$ was subjected to ethyl iodide and sodium carbonate in boiling methanol to afford pure $\mathbf{7 c}$ as described previously. ${ }^{17}$ 
When this method was transferred to the reaction of $7 \mathbf{a}$ with allyl bromide, $n$-butyl iodide, or benzyl bromide, the respective alkylation products $\mathbf{7 d}, \mathbf{7 e}$, and $\mathbf{7 f}$ were obtained as pure solids. Attempts to use other procedures for the synthesis of $\mathbf{7 d},{ }^{18} \mathbf{7 e},{ }^{19}$ and $\mathbf{7} \mathbf{f}^{20}$ led only to crude mixtures of starting compounds and products. Oxidation of 7c-f with the help of Jones reagent furnished the desired $N$-alkylated triacetonamines $\mathbf{3 c}-\mathbf{f}$ in moderate yields and high purity. These compounds were unequivocally characterized not only by elemental analyses but also by ${ }^{1} \mathrm{H}$ and ${ }^{13} \mathrm{C}$ NMR spectroscopic data for the first time.

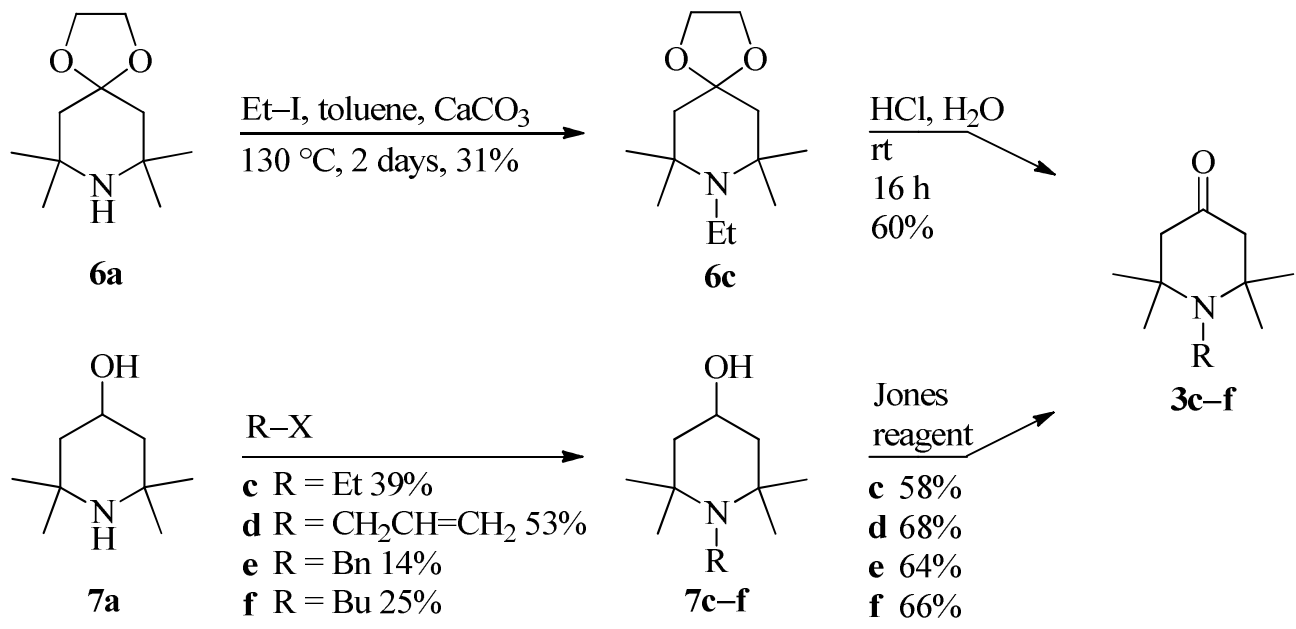

Scheme 2. Synthesis of $N$-alkylated triacetonamines 3c-f.

In subsequent investigations, we treated phorone (5) with ammonia (2a) and the primary amines 2b-f as described several times in the literature (Scheme 3). ${ }^{7 a, b, 10,11}$ Surprisingly, we observed a product with piperidine structure only in the reactions with ammonia (2a) and with methylamine (2b). Even in these cases, the ${ }^{1} \mathrm{H}$ and ${ }^{13} \mathrm{C}$ NMR spectra of the crude reaction mixtures indicated that not only the desired heterocycles $\mathbf{3 a}$ and $\mathbf{3 b}$ but also the unstable simple addition products $\mathbf{8 a}$ and $\mathbf{8 b}$ were formed. Whereas treatment of $\mathbf{5}$ with $\mathbf{2 a}$ led to the main product 3a and only small amounts of $\mathbf{8 a}$, the products $\mathbf{3 b}$ and $\mathbf{8 b}$ resulted from $\mathbf{5}$ and $\mathbf{2 b}$ in an approximate 3:2 ratio. Previously, in the unsuccessful attempt to methylate 3a with the help of formaldehyde and formic acid, ${ }^{12 a} \mathbf{8 b}$ was postulated to be an elusive intermediate to the final product phorone (5). When 5 was subjected to ethylamine (2c), we detected $\mathbf{8 c}$ as the only product in $80 \%$ yield. This result is contrary to an early report on the synthesis of isomeric 3c from 5 and 2c. In that report, ${ }^{7 a, b}$ however, the product could be characterized only by the melting point $\left(157-158{ }^{\circ} \mathrm{C}\right)$ and the elemental analysis of the corresponding chloroplatinate. When we prepared the hexachloroplatinates of $\mathbf{3 c}$ and $\mathbf{8 c}$, we obtained substances with melting points of 150-157 and $147-152{ }^{\circ} \mathrm{C}$, respectively. Although these melting points are relatively similar to each other and to the reported ${ }^{7 \mathrm{a}, \mathrm{b}}$ value, a mixture of both substances showed a significant 
melting point depression. Furthermore, NMR spectra indicated quite different compounds, which included a piperidin-4-one and an open-chain 6-aminohept-2-en-4-one structure.

On treatment of $\mathbf{5}$ with the amines $\mathbf{2 d}$, 2e, or $\mathbf{2 f}$, we got always mixtures of open-chain addition products 8 and 9 but no heterocycle of type 3. Thus, the reaction of allylamine (2d) afforded 8d (36\% yield) and 9d (36\%), whereas benzylamine (2e) led to 8e (29\%) and 9e (58\%), and $n$-butylamine (2f) gave $\mathbf{8 f}(31 \%)$ and $9 f(41 \%)$. The separation of the amines $\mathbf{8}$ and diamines 9 proved to be difficult. For example, vacuum distillation was not possible because both compounds tended to cleave off the corresponding primary amine, which resulted in the formation of $\mathbf{5}$. Nevertheless, we were able to characterize these open-chain addition products for the first time. In previous reports, the generation of open-chain adducts of $\mathbf{2 e}$ and $\mathbf{5}$ was postulated to explain byproducts and decay products in the supposed synthesis of $\mathbf{3 e}$ from benzylamine and phorone. ${ }^{7 \mathrm{a}, \mathrm{b}, 11}$ Our results indicate that it will be difficult to prepare $N$-alkylated triacetonamines from $\mathbf{5}$ and primary amines $\mathrm{RNH}_{2}$ if the substituents $\mathrm{R}$ are more bulky than methyl.

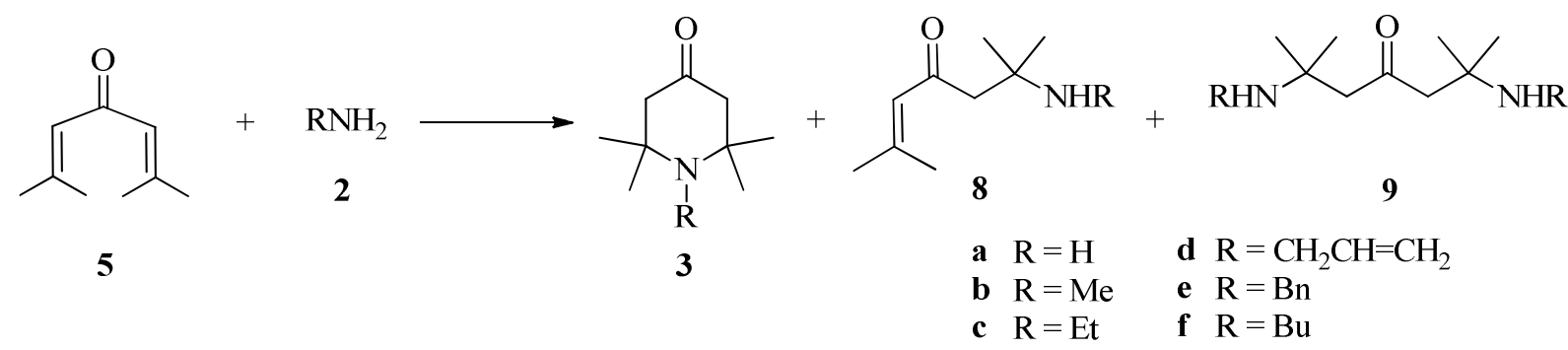

Scheme 3. Reactions of phorone (5) with ammonia (2a) or primary amines $\mathbf{2 b - f}$.

When we treated the neat parent compound 3a with ethyl iodide or allyl bromide at room temperature, we did not get the hydroiodide of $\mathbf{3 c}$ or the hydrobromide of $\mathbf{3 d}$ as it was described in literature. ${ }^{14}$ The only products, which could be identified, were the hydroiodide and the hydrobromide of 3a. After modification of the reaction conditions, we were able to synthesize 3d and 3e by alkylation of 3a. Thus, subjection of 3a to allyl bromide in hexane in the presence of potassium carbonate (rt, 7 days) afforded $\mathbf{3 d}$ in $6 \%$ yield, and $\mathbf{3 e}$ was formed analogically with $1 \%$ yield. The yields of the isolated pure products were very low because of incomplete conversion of 3a and loss of material during purification by chromatography. When similar conditions were used to treat 3a with ethyl iodide, we did not obtain any desired product $\mathbf{3 c}$.

The synthesis of triacetonamine (3a) from acetone (1) and ammonia (2a) can be transferred to the reaction of $\mathbf{1}$ with amine $\mathbf{2} \mathbf{b}$ to produce the heterocycle $\mathbf{3 b}$. If modern (heterogeneous) acidic catalysts $^{6 \mathrm{~b}, \mathrm{c}}$ are used, the conversion to generate $\mathbf{3 b}$ is similar to that leading to $\mathbf{3 a}$. However, the stability of $\mathbf{3 b}$ is significantly lower than that of $\mathbf{3 a}$. For example, $\mathbf{3} \mathbf{b}$ is degraded much more rapidly in boiling water than $\mathbf{3 a}$. These facts make it more difficult to isolate pure $\mathbf{3 b}$ 
from complex reaction mixtures. When we treated acetone (1) with benzylamine (2e) or $n$ butylamine (2f) in the presence of calcium chloride, we could not detect any heterocycles $\mathbf{3 e}$ or 3f, respectively, in the complicated mixtures of products with the help of ${ }^{1} \mathrm{H}$ and ${ }^{13} \mathrm{C} N M R$ spectroscopy. This outcome is in contrast to the literature ${ }^{9}$ but is plausible if compared with our results in connection with the reaction of phorone (5) with primary amines 2.

The low yields, observed in the alkylation reactions of 3a (see above), can lead to the assumption that the introduction of more bulky substituents at the $\mathrm{N}$ atom of $\mathbf{3 a}$ is always problematic. However, good yields can be achieved when quite different electrophiles are subjected to 3a. Highly reactive allenyl isothiocyanates like $\mathbf{1 0}$ are well known to produce a variety of thiazole derivatives when treated with nucleophiles (Scheme 4). ${ }^{21}$ Thus, triacetonamine (3a) was reacted with the cumulene $\mathbf{1 0}$ to furnish the $N$-arylpiperidin-4-one 11, and the secondary amine $4 \mathbf{a}$ was converted similarly to give the product $\mathbf{1 2}$. But even more sterically hindered amines, such as tert-amyl-tert-butylamine or bis(1,1-dimethylpropyl)amine, failed to give thiazole derivatives in the presence of isothiocyanate $\mathbf{1 0}$.

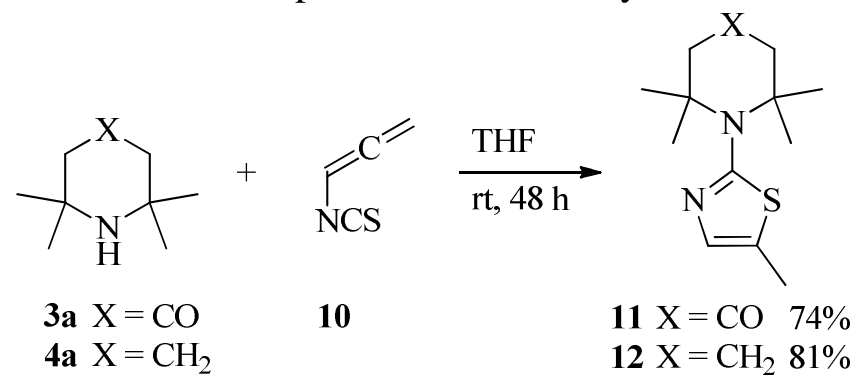

Scheme 4. Reactions of 2,2,6,6-tetramethylpiperidines with allenyl isothiocyanate (10).

\section{Conclusions}

With the help of the multi-step sequences via $6 \mathbf{a}$ and $\mathbf{6 c}$ or via $7 \mathbf{a}$ and $7 \mathbf{c}-\mathbf{f}$, it was possible for the first time to prepare the triacetone $N$-alkyl derivatives 3c-f (Scheme 2). The NMR data of $\mathbf{3 c}-\mathbf{f}$ were utilized to analyze whether these products were formed by treatment of phorone (5) with the corresponding primary amines $\mathbf{2 c - f}$ (Scheme 3). However, the addition products 8 and 9 were obtained instead of nitrogen heterocycles. Moreover, the reactions of acetone (1) with benzylamine (2e) or $n$-butylamine (2f) in the presence of calcium chloride did not lead to any 2,2,6,6-tetramethylpiperidin-4-one 3e or 3f, respectively (Scheme 1). Simple $N$-alkylation of the parent compound 3a proved also to be problematic if the substituent at the $\mathrm{N}$ atom is more bulky than methyl. Our results clarify some contradictory reports from literature. ${ }^{7 \mathrm{a}, \mathrm{b}, 9,11,14}$ Finally, we showed that $N$-arylation of triacetonamine (3a) is possible with good yield when the starting compound is treated with highly electrophilic allenyl isothiocyanate (10) to give the 2-aminothiazole derivative 11 (Scheme 4). 


\section{Experimental Section}

General. Melting points were determined with a Pentakon Dresden Boetius apparatus and were uncorrected. IR measurements were made on solutions in $\mathrm{KBr}$ cuvettes. ${ }^{1} \mathrm{H} \mathrm{NMR}$ and ${ }^{13} \mathrm{C}$ NMR spectra were recorded with Varian Unity Inova 400 spectrometer at 400 and $100.6 \mathrm{MHz}$, respectively. Elemental analyses were performed with a Vario EL elemental analyzer from Elementar Analysensysteme GmbH Hanau. Some of the amino compounds were transformed into their picrate salts by using standard procedures ${ }^{22}$ to get crystalline substances, which were appropriate for elemental analyses. Basic aluminum oxide and silica gel 60 (Macherey Nagel $\mathrm{GmbH} \& \mathrm{Co} . \mathrm{KG}$ ) were used for flash column chromatography. Mass spectra were determined with a Bruker micrOTOF-Q II 10228 spectrometer.

8-Ethyl-7,7,9,9-tetramethyl-1,4-dioxa-8-aza-spiro[4,5]decane (6c). A mixture of $\mathbf{6 a}^{16}(1.00 \mathrm{~g}$, $5.0 \mathrm{mmol})$, toluene $(10 \mathrm{~mL})$, ethyl iodide $(0.81 \mathrm{~mL}, 1.57 \mathrm{~g}, 10 \mathrm{mmol})$, and calcium carbonate $(0.65 \mathrm{~g}, 6.5 \mathrm{mmol})$ was heated in a glass ampoule at $130{ }^{\circ} \mathrm{C}$ for 2 days. After cooling and filtration, the solvent was removed at reduced pressure. The residue was washed with a small amount of cold $\left(0{ }^{\circ} \mathrm{C}\right)$ methanol to give $\mathbf{6 c}$.

6c. Colorless crystals, mp $49-52{ }^{\circ} \mathrm{C}, 0.35 \mathrm{~g}$, yield $31 \%$; IR $\left(v_{\max }, \mathrm{cm}^{-1}\right): 2915(\mathrm{CH}) .{ }^{1} \mathrm{H}$ NMR (400 MHz, $\left.\mathrm{CDCl}_{3}\right): \delta_{\mathrm{H}} 1.02\left(\mathrm{t},{ }^{3} \mathrm{~J}=7 \mathrm{~Hz}, 3 \mathrm{H}, \mathrm{NCH}_{2} \mathbf{C H}_{3}\right), 1.12$ (s, 12H, Me), $1.64(\mathrm{~s}, 4 \mathrm{H}$, $\left.\mathbf{C H}_{2} \mathrm{C}(\mathrm{Me})_{2}\right), 2.53$ (q, $\left.{ }^{3} \mathrm{~J}=7 \mathrm{~Hz}, 2 \mathrm{H}, \mathrm{NCH}_{2}\right), 3.92$ (s, 4H, OCH$)_{2}{ }^{13} \mathrm{C}$ NMR (100 MHz, CDCl 3 ): $\delta_{\mathrm{C}} 20.88$ (q, $\mathrm{NCH}_{2} \mathbf{C H}_{3}$ ), 27.83 (q, br, $\mathrm{Me}$ ), 37.23 (t, $\mathrm{NCH}_{2} \mathrm{CH}_{3}$ ), 47.54 (t, $\left.\mathbf{C H}_{\mathbf{2}} \mathrm{C}(\mathrm{Me})_{2}\right), 55.71$ (s, $\left.\mathrm{C}(\mathrm{Me})_{2}\right), 63.54$ (t, $\mathrm{OCH}_{2}$ ), 107.72 (s, OCO); Anal. Calcd for $\mathrm{C}_{13} \mathrm{H}_{25} \mathrm{NO}_{2}$ (227.34): C, 68.68; H, $11.08 ; \mathrm{N}, 6.16 \%$. Found $\mathrm{C}, 68.51 ; \mathrm{H}, 11.12 ; \mathrm{N}, 6.25 \%$.

1-Ethyl-2,2,6,6-tetramethylpiperidin-4-one (3c) from 6c. To a mixture of aqueous hydrogen chloride $(5 \%, 10 \mathrm{~mL})$ and $\mathrm{Et}_{2} \mathrm{O}(10 \mathrm{~mL})$ was added $\mathbf{6 c}(0.35 \mathrm{~g}, 1.50 \mathrm{mmol})$. After stirring for 16 $\mathrm{h}$ at room temperature, the mixture was alkalized and extracted three times with $\mathrm{Et}_{2} \mathrm{O}$. The combined organic layers were washed twice with saturated aqueous sodium chloride and with water. After drying with $\mathrm{MgSO}_{4}$, the solvent was removed at reduced pressure to give 3c.

3c. Yellow oil, $0.16 \mathrm{~g}$; yield 60\%, IR $\left(v_{\max }, \mathrm{cm}^{-1}\right): 2965(\mathrm{CH}), 1707(\mathrm{C}=\mathrm{O}) .{ }^{1} \mathrm{H}$ NMR $(400 \mathrm{MHz}$, $\mathrm{CDCl}_{3}$ ): $\delta_{\mathrm{H}} 1.10\left(\mathrm{t},{ }^{3} \mathrm{~J}=7 \mathrm{~Hz}, 3 \mathrm{H}, \mathrm{NCH}_{2} \mathbf{C H}_{3}\right), 1.12$ (s, 12H, Me), 2.33 (s, 4H, $\left.\mathbf{C H}_{2} \mathrm{C}(\mathrm{Me})_{2}\right), 2.58$ $\left(\mathrm{q},{ }^{3} \mathrm{~J}=7 \mathrm{~Hz}, 2 \mathrm{H}, \mathrm{NCH}_{2}\right) \cdot{ }^{13} \mathrm{C} \mathrm{NMR}\left(100 \mathrm{MHz}, \mathrm{CDCl}_{3}\right): \delta_{\mathrm{C}} 20.63\left(\mathrm{q}, \mathrm{NCH}_{2} \mathbf{C H}_{3}\right), 28.28(\mathrm{q}, \mathrm{br}$, $\mathrm{Me}$ ), 37.91 (t, $\mathrm{NCH}_{2} \mathrm{CH}_{3}$ ), 55.90 (t, $\left.\mathbf{C H}_{2} \mathrm{C}(\mathrm{Me})_{2}\right), 59.90$ (s, $\left.\mathbf{C}(\mathrm{Me})_{2}\right), 210.29$ (s, CO); picrate of 3c Anal. Calcd for $\mathrm{C}_{11} \mathrm{H}_{21} \mathrm{NO} \cdot \mathrm{C}_{6} \mathrm{H}_{9} \mathrm{~N}_{3} \mathrm{O}_{7}$ (418.20): C, 49.51; H, 5.87; N, 13.59\%. Found C, 48.97; H, 5.72; N, 13.74\%.

1-Benzyl-2,2,6,6-tetramethylpiperidin-4-ol (7e). We prepared 7 e from $7 \mathbf{a}^{12 \mathrm{a}}$ by using benzyl bromide and a known procedure, which was successful in the synthesis of $\mathbf{7 c} .{ }^{17}$ The product $7 \mathbf{e}$ was mentioned in literature, ${ }^{19}$ but spectroscopic data or the melting point were not published.

7e. White solid, mp $100{ }^{\circ} \mathrm{C}, 0.20 \mathrm{~g}$, yield $14 \% ;{ }^{1} \mathrm{H}$ NMR $\left(400 \mathrm{MHz}, \mathrm{CDCl}_{3}\right): \delta_{\mathrm{H}} 0.99(\mathrm{~s}, 6 \mathrm{H}, 2 \mathrm{x}$ $\mathrm{Me}$ ), 1.13 (s, 6H, 2 x Me), 1.52 (, ,t“, $\left.J=12 \mathrm{~Hz}, 2 \mathrm{H}, \mathbf{C H}_{2} \mathrm{CHOH}\right), 1.75$ (s, 1H, OH), 1.90 (dd, ${ }^{2} J$ $\left.=12 \mathrm{~Hz},{ }^{3} \mathrm{~J}=4.4 \mathrm{~Hz}, 2 \mathrm{H}, \mathbf{C H}_{2} \mathrm{CHOH}\right), 3.82\left(\mathrm{~s}, 2 \mathrm{H}, \mathrm{NCH}_{2}\right), 4.05(\mathrm{~m}, 1 \mathrm{H}, \mathbf{C H O H}), 7.44-7.15$ 
(m, 5H, ArH). ${ }^{13} \mathrm{C}$ NMR (100 MHz, $\left.\mathrm{CDCl}_{3}\right): \delta_{\mathrm{C}} 24.50$ (q, Me), 28.90 (q, Me), $50.12\left(\mathrm{t}, \mathbf{C H}_{2} \mathrm{CO}\right.$ ), 55.83 (s, C(Me) $)_{2}$ ), 64.32 (d, $\left.\mathbf{C H O H}\right), 125.87$ (d), 126.50 (d), 127.98 (d), 142.50 (s, Ar-C).

1-Butyl-2,2,6,6-tetramethylpiperidin-4-ol (7f). We synthesized 7f from 7a and $n$-butyl iodide by utilizing a known procedure, which was successful to prepare $\mathbf{7 c} .^{17}$ The product $\mathbf{7 f}$ was mentioned in literature, ${ }^{20}$ however, spectroscopic data were not given.

7f. White solid, mp $73{ }^{\circ} \mathrm{C}, 0.32 \mathrm{~g}$, yield $25 \%$; IR $\left(v_{\max }, \mathrm{cm}^{-1}\right): 3419(\mathrm{OH}), 2911(\mathrm{CH}) .{ }^{1} \mathrm{H}$ NMR (400 MHz, $\left.\mathrm{CDCl}_{3}\right): \delta_{\mathrm{H}} 0.90\left(\mathrm{t}, 3 \mathrm{H}, \mathrm{N}\left(\mathrm{CH}_{2}\right)_{3} \mathbf{C H}_{3},{ }^{3} J=7.4 \mathrm{~Hz}\right), 1.11(\mathrm{~s}, 6 \mathrm{H}, \mathrm{Me}), 1.20$ (,sext“", $\left.2 \mathrm{H}, \mathrm{N}\left(\mathrm{CH}_{2}\right)_{2} \mathbf{C H}_{2} \mathrm{CH}_{3},{ }^{3} J=7.4 \mathrm{~Hz}\right), 1.31$ (,„t“, $\left.J=12 \mathrm{~Hz}, 2 \mathrm{H}, \mathrm{CH}_{2} \mathrm{CH}(\mathrm{OH}), \mathrm{H}_{\mathrm{ax}}\right), 1.40-1.34$ (m, $\left.2 \mathrm{H}, \mathrm{NCH}_{2} \mathbf{C H}_{2}\right), 1.79\left(\mathrm{dd},{ }^{2} J=12 \mathrm{~Hz},{ }^{3} \mathrm{~J}=4 \mathrm{~Hz}, 2 \mathrm{H}, \mathbf{C H}_{2} \mathrm{CH}(\mathrm{OH}), \mathrm{H}_{\mathrm{eq}}\right), 2.35$ (t, 2H, NCH$)$, $3.94\left(\mathrm{tt},{ }^{3} J_{\mathrm{ax}}=12 \mathrm{~Hz},{ }^{3} J_{\text {eq }}=4 \mathrm{~Hz}, 1 \mathrm{H}, \mathbf{C H}(\mathrm{OH})\right) .{ }^{13} \mathrm{C} \mathrm{NMR}\left(100 \mathrm{MHz}, \mathrm{CDCl}_{3}\right): \delta_{\mathrm{C}} 14.09$ (q, $\left.\mathrm{N}\left(\mathrm{CH}_{2}\right)_{3} \mathbf{C H}_{3}\right), 20.55$ (t, $\left.\mathrm{N}\left(\mathrm{CH}_{2}\right)_{2} \mathbf{C H}_{2} \mathrm{CH}_{3}\right), 21.97$ (q, Me), 34.20 (q, Me), 37.99 (t, $\mathrm{NCH}_{2} \mathbf{C H}_{2}$ ), 43.97 (t, NCH$), 50.08$ (t, $\left.\mathbf{C H}_{2} \mathrm{CHOH}\right), 55.85$ (s, C(Me) $\left.)_{2}\right), 64.02$ (d, $\left.\mathbf{C H O H}\right)$.

1-Ethyl-2,2,6,6-tetramethylpiperidin-4-one (3c) from 7c. A solution of $7 \mathbf{c}^{17}(0.50 \mathrm{~g}, 2.7 \mathrm{mmol})$ in distilled acetone $(50 \mathrm{~mL})$ was flushed with nitrogen for $1 \mathrm{~h}$ and then cooled to $0{ }^{\circ} \mathrm{C}$. Jones reagent $\left(5.93 \mathrm{~mL}\right.$, prepared from $33.5 \mathrm{~g}$ of $\mathrm{CrO}_{3}$ and $130 \mathrm{~mL}$ of concd. $\mathrm{H}_{2} \mathrm{SO}_{4}$ filled up by water to a total volume of $250 \mathrm{~mL}$ ) was slowly added. After an additional reaction time of $1 \mathrm{~h}$, water $(50 \mathrm{~mL})$ was added, and the mixture was made alkaline and extracted with pentane $(5 \times)$. The combined organic layers were washed with saturated aqueous $\mathrm{NaHCO}_{3}(50 \mathrm{~mL})$ and $\mathrm{NaCl}(50$ $\mathrm{mL}$ ), and dried with $\mathrm{MgSO}_{4}$. After removal of the solvent under reduced pressure, 3c was isolated as a yellow oil ( $0.28 \mathrm{~g}, 58 \%$ yield), which was identical with $3 \mathbf{c}$ prepared from $\mathbf{6 c}$.

1-Allyl-2,2,6,6-tetramethylpiperidin-4-one (3d) from $\mathbf{7 d}$. This product was prepared from $\mathbf{7} \mathbf{d}^{18}$ $(0.50 \mathrm{~g}, 2.6 \mathrm{mmol})$ by oxidation with the help of Jones reagent as described for 3c. However, the reaction time was prolonged to $4 \mathrm{~h}$. The product $\mathbf{3 d}$ was stored under inert gas and with cooling.

3d. Yellow oil, $0.34 \mathrm{~g}$, yield 68\%; IR $\left(v_{\max }, \mathrm{cm}^{-1}\right): 2969(\mathrm{CH}), 1703(\mathrm{C}=\mathrm{O}), 1637(\mathrm{C}=\mathrm{C}) .{ }^{1} \mathrm{H}$ NMR (400 MHz, $\left.\mathrm{CDCl}_{3}\right): \delta_{\mathrm{H}} 1.12(\mathrm{~s}, 12 \mathrm{H}, \mathrm{Me}), 2.37$ (s, 4H, $\left.\mathbf{C H}_{2} \mathrm{C}(\mathrm{Me})_{2}\right), 3.23\left(\mathrm{dt},{ }^{3} \mathrm{~J}=5 \mathrm{~Hz},{ }^{4} \mathrm{~J}\right.$ $\left.=1.7 \mathrm{~Hz}, 2 \mathrm{H}, \mathrm{CH}_{2} \mathrm{CH}=\mathrm{CH}_{2}\right), 4.99-5.03\left(\mathrm{dq},{ }^{3} \mathrm{~J}=10 \mathrm{~Hz},{ }^{4} \mathrm{~J}=1.7 \mathrm{~Hz}, 1 \mathrm{H}, \mathrm{CH}=\mathrm{CH}_{2}\right), 5.19-5.25$ $\left(\mathrm{dq},{ }^{3} J_{\text {trans }}=17 \mathrm{~Hz},{ }^{4} \mathrm{~J}=1.7 \mathrm{~Hz}, 1 \mathrm{H}, \mathrm{CH}=\mathbf{C H}_{2}\right), 5.87-5.91\left(\mathrm{ddt},{ }^{3} J_{\text {trans }}=17 \mathrm{~Hz},{ }^{3} J_{\text {cis }}=10 \mathrm{~Hz},{ }^{3} \mathrm{~J}=\right.$ $\left.5 \mathrm{~Hz}, 1 \mathrm{H}, \mathrm{CH}_{2} \mathrm{CH}=\mathrm{CH}_{2}\right) .{ }^{13} \mathrm{C} \mathrm{NMR}\left(100 \mathrm{MHz}, \mathrm{CDCl}_{3}\right): \delta_{\mathrm{C}} 28.31(\mathrm{q}, \mathrm{br}, \mathrm{Me}), 46.28(\mathrm{t}$, $\left.\mathbf{C H}_{2} \mathbf{C H}=\mathrm{CH}_{2}\right), 55.83\left(\mathrm{t}, \mathbf{C O C H}{ }_{2}\right), 59.98\left(\mathrm{~s}, \mathbf{C}(\mathrm{Me})_{2}\right), 113.49(\mathrm{t}, \mathbf{C H}=\mathbf{C H}), 141.93$ (d, $\mathrm{CH}_{2} \mathbf{C H}=\mathrm{CH}_{2}$ ), 210.16 (s, CO); picrate of 3d Anal. Calcd for $\mathrm{C}_{12} \mathrm{H}_{21} \mathrm{NO} \mathrm{C}_{6} \mathrm{H}_{9} \mathrm{~N}_{3} \mathrm{O}_{7}$ (430.20): C, 50.94; H, 5.70; N, 13.20\%. Found C, 50.47; H, 5.48; N, 13.13\%.

1-Benzyl-2,2,6,6-tetramethylpiperidin-4-one (3e) from 7e. This product was synthesized from $7 \mathbf{e}^{19}(0.20 \mathrm{~g}, 0.80 \mathrm{mmol})$ by oxidation with Jones reagent as described for $3 \mathbf{d}$.

3e. Colorless crystals, mp $102{ }^{\circ} \mathrm{C}, 0.13 \mathrm{~g}$, yield 64\%; IR ( $\left.v_{\max }, \mathrm{cm}^{-1}\right): 3081(\mathrm{C}=\mathrm{C}), 2973(\mathrm{CH})$, $1707(\mathrm{C}=\mathrm{O}) .{ }^{1} \mathrm{H}$ NMR $\left(400 \mathrm{MHz}, \mathrm{CDCl}_{3}\right): \delta_{\mathrm{H}} 1.13$ (s, 12H, Me), 2.47 (s, 4H, $\left.\mathbf{C H}_{2} \mathrm{C}(\mathrm{Me})_{2}\right), 3.91$ (s, 2H, $\left.\mathbf{C H}_{2} \mathrm{Ph}\right), 7.17-7.49(\mathrm{~m}, 5 \mathrm{H}, \mathrm{ArH}) .{ }^{13} \mathrm{C} \mathrm{NMR}\left(100 \mathrm{MHz}, \mathrm{CDCl}_{3}\right): \delta_{\mathrm{C}} 28.33$ (q, br, Me), 47.52 (t, $\mathbf{C H}_{2} \mathrm{Ph}$ ), 55.89 (t, $\mathbf{C H}_{2} \mathrm{CO}$ ), 60.05 (s, $\mathbf{C}(\mathrm{Me})_{2}$ ), 125.88 (d), 126.52 (d), 128.00 (d), 144.58 (s, ArC), 210.01 (s, CO); Anal. Calcd for $\mathrm{C}_{16} \mathrm{H}_{23} \mathrm{NO}$ (245.18): C, 78.32; H, 9.45; N, 5.71\%. Found C, 77.77; H, 9.38; N, 5.81\%. 
1-Butyl-2,2,6,6-tetramethylpiperidin-4-one (3f). This product was prepared from $7 \mathbf{f}^{20}(0.50 \mathrm{~g}$, $2.3 \mathrm{mmol}$ ) by oxidation with Jones reagent as described for $\mathbf{3 c}$. However, the reaction time was prolonged to $6 \mathrm{~h}$. The product $\mathbf{3 f}$ was stored under inert gas and with cooling.

3f. Yellow oil, $0.32 \mathrm{~g}$, yield 66\%; IR $\left(v_{\max }, \mathrm{cm}^{-1}\right)$ : $2968(\mathrm{CH}), 1708(\mathrm{C}=\mathrm{O}) .{ }^{1} \mathrm{H}$ NMR (400 MHz, $\left.\mathrm{CDCl}_{3}\right): \delta_{\mathrm{H}} 0.91\left(\mathrm{t}, 3 \mathrm{H}, \mathrm{N}\left(\mathrm{CH}_{2}\right)_{3} \mathbf{C H}_{3},{ }^{3} \mathrm{~J}=7.4 \mathrm{~Hz}\right), 1.11(\mathrm{~s}, 12 \mathrm{H}, \mathrm{Me}), 1.25$ (,sext“, $2 \mathrm{H}$, $\left.\mathrm{N}\left(\mathrm{CH}_{2}\right)_{2} \mathbf{C H}_{2} \mathrm{CH}_{3},{ }^{3} \mathrm{~J}=7.4 \mathrm{~Hz}\right), 1.43-1.51\left(\mathrm{~m}, 2 \mathrm{H}, \mathrm{NCH}_{2} \mathbf{C H}_{2}\right), 2.32\left(\mathrm{~s}, 4 \mathrm{H}, \mathbf{C H}_{2} \mathrm{C}(\mathrm{Me})_{2}\right), 2.44$ $\left(\mathrm{m}, 2 \mathrm{H}, \mathrm{NCH}_{2}\right) .{ }^{13} \mathrm{C} \mathrm{NMR}\left(100 \mathrm{MHz}, \mathrm{CDCl}_{3}\right): \delta_{\mathrm{C}} 14.05$ (q, $\left.\mathrm{N}\left(\mathrm{CH}_{2}\right)_{3} \mathbf{C H}_{3}\right), 20.55$ (t, $\left.\mathrm{N}\left(\mathrm{CH}_{2}\right)_{2} \mathbf{C H}_{2} \mathrm{CH}_{3}\right), 28.27$ (q, br, Me), 37.75 (t, $\left.\mathrm{NCH}_{2} \mathbf{C H}_{2}\right), 44.35$ (t, NCH$), 55.87$ (t, $\mathrm{COCH}_{2}$ ), 59.82 (s, C(Me) 2 ), 210.26 (s, CO); picrate of 3f Anal. Calcd for $\mathrm{C}_{13} \mathrm{H}_{25} \mathrm{NO} \cdot \mathrm{C}_{6} \mathrm{H}_{3} \mathrm{~N}_{3} \mathrm{O}_{7}$ (440.19): C, 51.81; H, 6.41; N, 12.72\%. Found C, 51.89; H, 6.42; N, $12.72 \%$.

6-Amino-2,6-dimethylhept-2-en-4-one (8a). To an aqueous solution of ammonia (2a) (19\%, 22 $\mathrm{mL})$ was added phorone $(5)(7.8 \mathrm{~mL}, 6.90 \mathrm{~g}, 50 \mathrm{mmol})$. The mixture was stirred at room temperature for $48 \mathrm{~h}$ until a clear yellow solution had been formed, which was acidified with aqueous hydrogen chloride $\left(0.5\right.$ molar) and washed with $\mathrm{Et}_{2} \mathrm{O}(3 \times)$. The aqueous solution was alkalized with the help of aqueous potassium hydroxide (6 molar) and extracted with $\mathrm{Et}_{2} \mathrm{O}(3 \times)$. The combined organic layers were dried with $\mathrm{MgSO}_{4}$. After removal of the solvent at reduced pressure, a solid $(1.51 \mathrm{~g}, 19 \%)$ remained. ${ }^{1} \mathrm{H}$ NMR spectra indicated that 3a and 8a were formed in an approximate 13:1 ratio.

8a. ${ }^{1} \mathrm{H}$ NMR (400 MHz, $\left.\mathrm{CDCl}_{3}\right)$ : $\delta_{\mathrm{H}} 1.22\left(\mathrm{~s}, 6 \mathrm{H}, \mathrm{CH}_{2} \mathrm{C}(\mathbf{M e})_{2}\right), 1.86\left(\mathrm{~s}, 3 \mathrm{H}, \mathrm{CH}=\mathrm{C}(\mathbf{M e})_{2}\right), 2.12$ (s, 3H, CH=C(Me) $\left.)_{2}\right), 2.52$ (s, 2H, $\left.\mathbf{C H}_{2} \mathrm{C}(\mathrm{Me})_{2}\right), 6.02$ (br. s, $\left.1 \mathrm{H}, \mathbf{C H}=\mathrm{C}(\mathrm{Me})_{2}\right) .{ }^{13} \mathrm{C} \mathrm{NMR}(100$ $\mathrm{MHz} \mathrm{CDCl}_{3}$ ): $\delta_{\mathrm{C}} 20.58$ (q, $\mathrm{CH}=\mathrm{C}(\mathbf{M e})_{2}$ ), 27.61 (q, $\left.\mathrm{CH}_{2} \mathrm{C}(\mathbf{M e})_{2}\right), 30.47$ (q, $\left.\mathrm{CH}=\mathrm{C}(\mathbf{M e})_{2}\right), 49.06$ (s, $\left.\mathrm{CH}_{2} \mathbf{C}(\mathrm{Me})_{2}\right), 56.06$ ( $\left.\mathrm{t}, \mathbf{C H}_{2} \mathrm{C}(\mathrm{Me})_{2}\right), 124.87$ (d, $\left.\mathbf{C H}=\mathrm{C}(\mathrm{Me})_{2}\right), 155.44$ (s, $\left.\mathrm{CH}=\mathbf{C}(\mathrm{Me})_{2}\right)$, 200.43 (s, C=O).

2,6-Dimethyl-6-methylaminohept-2-en-4-one (8b). To an aqueous solution of $\mathbf{2 b}(4.3 \mathrm{~mL}$, $40 \%)$ was added $5(7.8 \mathrm{~mL}, 6.90 \mathrm{~g}, 50 \mathrm{mmol})$. The mixture was stirred at room temperature for $48 \mathrm{~h}$. Thereafter, workup was performed as described for the reaction of $\mathbf{2 a}$ with $\mathbf{5}$. In this case, a mixture (2.21 g, 25\%) of the products $\mathbf{3 b}$ and $\mathbf{8 b}$ with an approximate 3:2 ratio was formed. When the workup was conducted with aqueous $\mathrm{H}_{2} \mathrm{SO}_{4}(15 \%)$ instead of aqueous $\mathrm{HCl}(0.5$ molar), the proportion of $\mathbf{8 b}$ was significantly lower whereas the yield of $\mathbf{3 b}$ (15\%) was nearly unchanged.

8b. Yellow oil, $0.88 \mathrm{~g}$, yield 10\%; ${ }^{1} \mathrm{H}$ NMR (400 MHz, $\left.\mathrm{CDCl}_{3}\right): \delta_{\mathrm{H}} 0.99\left(\mathrm{~s}, 6 \mathrm{H}, \mathrm{CH}_{2} \mathrm{C}(\mathbf{M e})_{2}\right)$, $1.73\left(\mathrm{~s}, 3 \mathrm{H}, \mathrm{CH}=\mathrm{C}(\mathbf{M e})_{2}\right), 1.98\left(\mathrm{~s}, 3 \mathrm{H}, \mathrm{CH}=\mathrm{C}(\mathbf{M e})_{2}\right), 2.13$ (s, 3H, NMe), 2.36 (s, 2H, $\left.\mathbf{C H}_{2} \mathrm{C}(\mathrm{Me})_{2}\right), 5.90\left(\mathrm{~s}, 1 \mathrm{H}, \mathbf{C H}=\mathrm{C}(\mathrm{Me})_{2}\right) \cdot{ }^{13} \mathrm{C} \mathrm{NMR}\left(100 \mathrm{MHz}, \mathrm{CDCl}_{3}\right): \delta_{\mathrm{C}} 20.35$ (q, $\left.\mathrm{CH}=\mathrm{C}(\mathbf{M e})_{2}\right), 26.54$ (q, $\left.\mathrm{CH}_{2} \mathrm{C}(\mathbf{M e})_{2}\right), 27.41$ (q, $\left.\mathrm{CH}=\mathrm{C}(\mathbf{M e})_{2}\right), 28.46$ (q, NMe), 51.42 (t, $\left.\mathbf{C H}_{2} \mathrm{C}(\mathrm{Me})_{2}\right), 52.71\left(\mathrm{~s}, \mathrm{CH}_{2} \mathbf{C}(\mathrm{Me})_{2}\right), 124.89$ (d, $\left.\mathbf{C H}=\mathrm{C}(\mathrm{Me})_{2}\right), 154.91$ (s, $\left.\mathrm{CH}=\mathbf{C}(\mathrm{Me})_{2}\right), 200.45$ (s, CO).

6-Ethylamino-2,6-dimethylhept-2-en-4-one (8c). To a mixture of $\mathbf{2 c}(3.31 \mathrm{~mL}, 2.25 \mathrm{~g}, 50$ $\mathrm{mmol})$ and water $(5 \mathrm{~mL})$ was added $5(7.8 \mathrm{~mL}, 6.90 \mathrm{~g}, 50 \mathrm{mmol})$. After stirring at room temperature for $48 \mathrm{~h}$, the reaction mixture was acidified with aqueous $\mathrm{H}_{2} \mathrm{SO}_{4}(15 \%)$ and washed with $\mathrm{Et}_{2} \mathrm{O}(3 \times)$. The aqueous solution was made alkaline with aqueous potassium hydroxide (6 
molar) and extracted with $\mathrm{Et}_{2} \mathrm{O}(3 \times)$. The combined organic layers were dried with $\mathrm{MgSO}_{4}$ to give the product $8 \mathbf{c}$ after removal of the solvent at reduced pressure.

8c. Yellow oil, $7.32 \mathrm{~g}$, yield 80\%; IR $\left(v_{\max }, \mathrm{cm}^{-1}\right)$ : $3330(\mathrm{NH}), 2967(\mathrm{CH}), 1675(\mathrm{CO}) .{ }^{1} \mathrm{H}$ NMR $\left(400 \mathrm{MHz}, \mathrm{CDCl}_{3}\right): \delta_{\mathrm{H}} 1.08\left(\mathrm{t},{ }^{3} \mathrm{~J}=7 \mathrm{~Hz}, 3 \mathrm{H}, \mathrm{NCH}_{2} \mathbf{C H}_{3}\right), 1.13\left(\mathrm{~s}, 6 \mathrm{H}, \mathrm{CH}_{2} \mathrm{C}(\mathbf{M e})_{2}\right), 1.86(\mathrm{~s}, 3 \mathrm{H}$, $\left.\mathrm{CH}=\mathrm{C}(\mathbf{M e})_{2}\right), 2.11\left(\mathrm{~s}, 3 \mathrm{H}, \mathrm{CH}=\mathrm{C}(\mathbf{M e})_{2}\right), 2.51\left(\mathrm{~s}, 2 \mathrm{H}, \mathbf{C H}_{2} \mathrm{C}(\mathrm{Me})_{2}\right), 2.55\left(\mathrm{q},{ }^{3} \mathrm{~J}=7 \mathrm{~Hz}, 2 \mathrm{H}\right.$, $\left.\mathrm{NCH}_{2}\right) 6.03\left(\mathrm{~s}, 1 \mathrm{H}, \mathbf{C H}=\mathrm{C}(\mathrm{Me})_{2}\right) .{ }^{13} \mathrm{C} \mathrm{NMR}\left(100 \mathrm{MHz}, \mathrm{CDCl}_{3}\right): \delta_{\mathrm{C}} 15.73\left(\mathrm{q}, \mathrm{NCH}_{2} \mathbf{C H}_{3}\right), 20.64$ (q, $\left.\mathrm{CH}=\mathrm{C}(\mathbf{M e})_{2}\right), 27.48$ (q, $\left.\mathrm{CH}_{2} \mathrm{C}(\mathbf{M e})_{2}\right), 27.69$ (q, $\left.\mathrm{CH}=\mathrm{C}(\mathbf{M e})_{2}\right), 36.42\left(\mathrm{t}, \mathrm{NCH}_{2}\right), 52.15$ (t, $\left.\mathbf{C H}_{2} \mathrm{C}(\mathrm{Me})_{2}\right), 53.08$ (s, $\left.\mathrm{CH}_{2} \mathbf{C}(\mathrm{Me})_{2}\right), 125.22$ (d, $\left.\mathbf{C H}=\mathrm{C}(\mathrm{Me})_{2}\right), 155.13$ (s, $\left.\mathrm{CH}=\mathbf{C}(\mathrm{Me})_{2}\right), 200.87$ (s, CO). HRMS: $\mathrm{C}_{11} \mathrm{H}_{21} \mathrm{NO}$ requires $m / z$ 184.1696. Found 184.1688.

6-Allylamino-2,6-dimethylhept-2-en-4-one (8d) and 2,6-Bis-allylamino-2,6-dimethyl heptan-4-one (9d). A mixture of $\mathbf{2 d}(3.74 \mathrm{~mL}, 2.85 \mathrm{~g}, 50 \mathrm{mmol})$ and water $(5 \mathrm{~mL})$ was treated with $5(7.8 \mathrm{~mL}, 6.90 \mathrm{~g}, 50 \mathrm{mmol})$ as described for the reaction of $\mathbf{2 c}$ with $\mathbf{5}$. In this case, a mixture $(5.77 \mathrm{~g})$ of the products $8 \mathbf{d}$ and $9 \mathbf{d}$ was obtained. ${ }^{1} \mathrm{H}$ NMR spectra indicated that $\mathbf{8 d}$ and 9d were formed in an approximate $2: 1$ ratio.

8d. Yellow oil, $3.50 \mathrm{~g}$, yield 36\%; ${ }^{1} \mathrm{H}$ NMR (400 MHz, $\left.\mathrm{CDCl}_{3}\right): \delta_{\mathrm{H}} 1.12\left(\mathrm{~s}, 6 \mathrm{H}, \mathrm{CH}_{2} \mathrm{C}(\mathbf{M e})_{2}\right)$, 1.84 (br. s, 3H, CH=C(Me) $\left.)_{2}\right), 2.12$ (s, 3H, $\left.\mathrm{CH}=\mathrm{C}(\mathbf{M e})_{2}\right), 2.50\left(\mathrm{~s}, 2 \mathrm{H}, \mathbf{C H}_{2} \mathrm{C}(\mathrm{Me})_{2}\right), 3.13$ (dt, ${ }^{3} \mathrm{~J}=$ $\left.5 \mathrm{~Hz},{ }^{4} \mathrm{~J}=1.7 \mathrm{~Hz}, 2 \mathrm{H}, \mathbf{C H}_{2} \mathrm{CH}=\mathrm{CH}_{2}\right), 5.02\left(\mathrm{dq},{ }^{3} \mathrm{~J}=10 \mathrm{~Hz},{ }^{4} \mathrm{~J}=1.7 \mathrm{~Hz}, 1 \mathrm{H}, \mathbf{C H}=\mathrm{CH}_{2}\right), 5.14(\mathrm{dq}$, $\left.{ }^{3} J_{\text {trans }}=17 \mathrm{~Hz},{ }^{4} \mathrm{~J}=1.7 \mathrm{~Hz}, 2 \mathrm{H}, \mathrm{CH}=\mathbf{C H}_{2}\right), 5.87\left(\mathrm{ddt},{ }^{3} J_{\text {trans }}=17 \mathrm{~Hz},{ }^{3} J_{\text {cis }}=10 \mathrm{~Hz},{ }^{3} \mathrm{~J}=5 \mathrm{~Hz}, 1 \mathrm{H}\right.$, $\mathrm{CH}_{2} \mathbf{C H}=\mathrm{CH}_{2}$ ), 6.00 (br. s, $\left.1 \mathrm{H},-\mathbf{C H}=\mathrm{C}(\mathrm{Me})_{2}\right) .{ }^{13} \mathrm{C} \mathrm{NMR}\left(100 \mathrm{MHz}, \mathrm{CDCl}_{3}\right): \delta_{\mathrm{C}} 20.43(\mathrm{q}$, $\left.\mathrm{CH}=\mathrm{C}(\mathbf{M e})_{2}\right), 27.12$ (q, $\left.\mathrm{CH}_{2} \mathrm{C}(\mathbf{M e})_{2}\right), 45.23\left(\mathrm{t}, \mathbf{C H}_{2} \mathrm{CH}=\mathrm{CH}_{2}\right), 52.94\left(\mathrm{~s}, \mathrm{CH}_{2} \mathbf{C}(\mathrm{Me})_{2}\right), 53.10$ (t, $\left.\mathbf{C H}_{2} \mathrm{C}(\mathrm{Me})_{2}\right), 115.40$ (t, $\left.\mathbf{C H}=\mathbf{C H}_{2}\right) ; 126.07$ (d, $\left.\mathbf{C H}=\mathrm{C}(\mathrm{Me})_{2}\right), 137.23\left(\mathrm{~d}, \mathrm{CH}_{2} \mathbf{C H}=\mathrm{CH}_{2}\right), 155.07$ $\left(\mathrm{s}, \mathrm{CH}=\mathbf{C}(\mathrm{Me})_{2}\right), 200.57$ (s, C=O). HRMS: $\mathrm{C}_{12} \mathrm{H}_{21} \mathrm{NO}$ requires $m / z$ 196.1690. Found 196.1696.

9d. Yellow oil, $2.27 \mathrm{~g}$, yield 36\%; ${ }^{1} \mathrm{H}$ NMR (400 MHz, $\left.\mathrm{CDCl}_{3}\right): \delta_{\mathrm{H}} 1.11\left(\mathrm{~s}, 12 \mathrm{H}, \mathrm{CH}_{2} \mathrm{C}(\mathbf{M e})_{2}\right)$, $2.50\left(\mathrm{~s}, 4 \mathrm{H}, \mathbf{C H}_{2} \mathrm{C}(\mathrm{Me})_{2}\right), 3.10\left(\mathrm{dt},{ }^{3} \mathrm{~J}=5 \mathrm{~Hz},{ }^{4} \mathrm{~J}=1.7 \mathrm{~Hz}, 4 \mathrm{H}, \mathbf{C H}_{2} \mathrm{CH}=\mathrm{CH}_{2}\right), 5.02$ (dq, ${ }^{3} J=10$ $\left.\mathrm{Hz},{ }^{4} \mathrm{~J}=1.7 \mathrm{~Hz}, 2 \mathrm{H}, \mathbf{C H}=\mathrm{CH}_{2}\right), 5.14\left(\mathrm{dq},{ }^{3} J_{\text {trans }}=17 \mathrm{~Hz},{ }^{4} \mathrm{~J}=1.7 \mathrm{~Hz}, 4 \mathrm{H}, \mathbf{C H}=\mathbf{C H}_{2}\right), 5.87$ (ddt, $\left.{ }^{3} J_{\text {trans }}=17 \mathrm{~Hz},{ }^{3} J_{\text {cis }}=10 \mathrm{~Hz},{ }^{3} \mathrm{~J}=5 \mathrm{~Hz}, 2 \mathrm{H}, \mathrm{CH}_{2} \mathbf{C H}=\mathrm{CH}_{2}\right) .{ }^{13} \mathrm{C} \mathrm{NMR}\left(100 \mathrm{MHz}, \mathrm{CDCl}_{3}\right): 27.65$ (q, $\left.\mathrm{CH}_{2} \mathrm{C}(\mathbf{M e})_{2}\right), 52.51$ (s, $\left.\mathrm{CH}_{2} \mathbf{C}(\mathrm{Me})_{2}\right), 52.53$ (t, $\left.\mathbf{C H}_{2} \mathrm{C}(\mathrm{Me})_{2}\right), 115.40$ (t, $\mathbf{C H}=\mathbf{C H}_{2}$ ); 137.13 (d, $\left.\mathrm{CH}_{2} \mathbf{C H}=\mathrm{CH}_{2}\right), 210.86\left(\mathrm{~s}, \mathbf{C}=\mathrm{O}\right.$ ). picrate of 9d Anal. Calcd for $\mathrm{C}_{15} \mathrm{H}_{28} \mathrm{~N}_{2} \mathrm{O} \cdot \mathrm{C}_{6} \mathrm{H}_{3} \mathrm{~N}_{3} \mathrm{O}_{7}$ (487.26): C, 45.64; H, 4.82; N, 15.77\%. Found C, 45.33; H, 4.78; N, $12.72 \%$.

6-Benzylamino-2,6-dimethylhept-2-en-4-one (8e) and 2,6-Bis-benzylamino-2,6-dimethyl heptan-4-one (9e). A mixture of $2 \mathrm{e}(5.46 \mathrm{~mL}, 5.36 \mathrm{~g}, 50 \mathrm{mmol})$ and water $(5 \mathrm{~mL})$ was treated with $5(7.8 \mathrm{~mL}, 6.90 \mathrm{~g}, 50 \mathrm{mmol})$ as described for the reaction of $\mathbf{2 c}$ with $\mathbf{5}$. In this case, a mixture $(8.63 \mathrm{~g})$ of the products $8 \mathbf{e}$ and $9 \mathbf{e}$ was obtained. ${ }^{1} \mathrm{H}$ NMR spectra indicated that $8 \mathbf{e}$ and 9e were formed in an approximate 1:1 ratio.

8e. Yellow oil, $3.53 \mathrm{~g}$, yield 29\%; ${ }^{1} \mathrm{H}$ NMR (400 MHz, $\left.\mathrm{CDCl}_{3}\right): \delta_{\mathrm{H}} 1.18\left(\mathrm{~s}, 6 \mathrm{H}, \mathrm{CH}_{2} \mathrm{C}(\mathbf{M e})_{2}\right)$, 1.84 (s, 3H, $\left.\mathrm{CH}=\mathrm{C}(\mathbf{M e})_{2}\right), 2.03$ (br. s, 1H, NH), 2.09 (s, 3H, $\left.\mathrm{CH}=\mathrm{C}(\mathbf{M e})_{2}\right), 2.58$ (s, 2H, $\mathbf{C H}_{2} \mathrm{C}(\mathrm{Me})_{2}$ ), 3.63 (s, 2H, $\mathbf{C H}_{2} \mathrm{Ph}$ ), 6.01 (br. s, $\left.1 \mathrm{H}, \mathbf{C H}=\mathrm{C}(\mathrm{Me})_{2}\right), 7.17-7.49$ (m, 5H, ArH). ${ }^{13} \mathrm{C}$ NMR (100 MHz, $\left.\mathrm{CDCl}_{3}\right): \delta_{\mathrm{C}} 20.57\left(\mathrm{q}, \mathrm{CH}=\mathrm{C}(\mathbf{M e})_{2}\right), 27.57$ (q, $\left.\mathrm{CH}_{2}-\mathrm{C}(\mathbf{M e})_{2}\right), 27.62$ (q, $\left.\mathrm{CH}=\mathrm{C}(\mathbf{M e})_{2}\right), 46.32\left(\mathrm{t}, \mathbf{C H}_{2} \mathrm{Ph}\right), 52.30\left(\mathrm{t}, \mathbf{C H}_{2} \mathrm{C}(\mathrm{Me})_{2}\right), 52.97\left(\mathrm{~s}, \mathrm{CH}_{2} \mathbf{C}(\mathrm{Me})_{2}\right), 125.17(\mathrm{~d}$, 
$\left.\mathbf{C H}=\mathrm{C}(\mathrm{Me})_{2}\right), 140.68(\mathrm{~s}, \mathrm{ArC}), 155.00\left(\mathrm{~s}, \mathrm{CH}=\mathbf{C}(\mathrm{Me})_{2}\right), 200.58$ (s, CO). HRMS: $\mathrm{C}_{16} \mathrm{H}_{23} \mathrm{NO}$ requires $m / z$ 246.1852. Found 246.1852.

9e. Yellow oil, $5.10 \mathrm{~g}$, yield 58\%; ${ }^{1} \mathrm{H} \mathrm{NMR}\left(400 \mathrm{MHz}, \mathrm{CDCl}_{3}\right): \delta_{\mathrm{H}} 1.19\left(\mathrm{~s}, 12 \mathrm{H}, \mathrm{CH}_{2} \mathrm{C}(\mathrm{Me})_{2}\right)$, 2.57 (s, 4H, $\left.\mathbf{C H}_{2} \mathrm{C}(\mathrm{Me})_{2}\right), 3.67$ (s, 4H, $\left.\mathbf{C H}_{2} \mathrm{Ph}\right), 7.16-7.33$ (m, 10H, ArH). ${ }^{13} \mathrm{C}$ NMR $(100 \mathrm{MHz}$, $\left.\mathrm{CDCl}_{3}\right): \delta_{\mathrm{C}} 27.45\left(\mathrm{q}, \mathrm{CH}_{2} \mathrm{C}(\mathbf{M e})_{2}\right), 46.81\left(\mathrm{t}, \mathbf{C H}_{2} \mathrm{Ph}\right), 52.30\left(\mathrm{t}, \mathbf{C H}_{2} \mathrm{C}(\mathrm{Me})_{2}\right), 52.89$ (s, $\mathrm{CH}_{2} \mathrm{C}(\mathrm{Me})_{2}$ ), 140.68 (s, ArC), 210.91 (s, CO). HRMS: $\mathrm{C}_{23} \mathrm{H}_{32} \mathrm{~N}_{2} \mathrm{O}$ requires $m / z$ 353.2574. Found 353.2587.

6-Butylamino-2,6-dimethylhept-2-en-4-one (8f) and 2,6-Bis-butylamino-2,6-dimethyl heptan-4-one (9f). A mixture of $\mathbf{2 f}(4.94 \mathrm{~mL}, 3.66 \mathrm{~g}, 50 \mathrm{mmol})$ and water $(5 \mathrm{~mL})$ was treated with $5(7.8 \mathrm{~mL}, 6.90 \mathrm{~g}, 50 \mathrm{mmol})$ as described for the reaction of $\mathbf{2 c}$ with $\mathbf{5}$. In this case, a mixture $(6.10 \mathrm{~g})$ of the products $8 \mathbf{8}$ and $9 \mathbf{f}$ was obtained. ${ }^{1} \mathrm{H}$ NMR spectra indicated that $8 \mathbf{f}$ and $9 \mathbf{f}$ were formed in an approximate 3:2 ratio.

8f. Yellow oil, $3.22 \mathrm{~g}$, yield 31\%; ${ }^{1} \mathrm{H}$ NMR (400 $\left.\mathrm{MHz}, \mathrm{CDCl}_{3}\right): \delta_{\mathrm{H}} 0.87\left(\mathrm{t}, 3 \mathrm{H}, \mathrm{N}\left(\mathrm{CH}_{2}\right)_{3} \mathbf{C H}_{3},{ }^{3} \boldsymbol{J}\right.$ $=7 \mathrm{~Hz}), 1.10\left(\mathrm{~s}, 6 \mathrm{H}, \mathrm{CH}_{2} \mathrm{C}(\mathbf{M e})_{2}\right), 1.32\left(\mathrm{~m}, 2 \mathrm{H}, \mathrm{N}\left(\mathrm{CH}_{2}\right)_{2} \mathbf{C H}_{2} \mathrm{CH}_{3}\right), 1.40\left(\mathrm{~m}, 2 \mathrm{H}, \mathrm{NCH}_{2} \mathbf{C H}_{2}\right)$, 1.68 (br. s, 1H, NH), 1.84 (s, 3H, CH=C(Me $\left.)_{2}\right), 2.09$ (s, 3H, $\left.\mathrm{CH}=\mathrm{C}(\mathbf{M e})_{2}\right), 2.44$ (m, 2H, NCH$)$, 2.48 (s, 2H, $\left.\mathbf{C H}_{2} \mathrm{C}(\mathrm{Me})_{2}\right), 6.02$ (br. s, $\left.1 \mathrm{H}, \mathbf{C H}=\mathrm{C}(\mathrm{Me})_{2}\right) .{ }^{13} \mathrm{C} \mathrm{NMR}\left(100 \mathrm{MHz}, \mathrm{CDCl}_{3}\right): \delta_{\mathrm{C}} 13.99$ (q, $\left.\mathrm{N}\left(\mathrm{CH}_{2}\right)_{3} \mathbf{C H}_{3}\right), 20.59$ (t, $\left.\mathrm{N}\left(\mathrm{CH}_{2}\right)_{2} \mathbf{C H}_{2} \mathrm{CH}_{3}\right), 27.27$ (q, $\left.\mathrm{CH}=\mathrm{C}(\mathbf{M e})_{2}\right), 27.54$ (q, $\left.\mathrm{CH}_{2} \mathrm{C}(\mathbf{M e})_{2}\right)$, 32.90 (t, $\left.\mathrm{NCH}_{2} \mathbf{C H}_{2}\right), 41.94$ (t, $\left.\mathrm{NCH}_{2}\right), 52.32$ ( $\left.\mathrm{s}, \mathrm{CH}_{2} \mathbf{C}(\mathrm{Me})_{2}\right), 52.40$ (t, $\left.\mathbf{C H}_{\mathbf{2}} \mathrm{C}(\mathrm{Me})_{2}\right), 125.29$ (d, $\left.\mathbf{C H}=\mathrm{C}(\mathrm{Me})_{2}\right), 154.75 \quad\left(\mathrm{~s}, \mathrm{CH}=\mathbf{C}(\mathrm{Me})_{2}\right), 200.85$ (s, CO). HRMS: $\mathrm{C}_{13} \mathrm{H}_{25} \mathrm{NO}$ requires $\mathrm{m} / z$ 212.2007. Found 212.2009.

9f. Yellow oil, $2.88 \mathrm{~g}$, yield 41\%; ${ }^{1} \mathrm{H}$ NMR (400 $\left.\mathrm{MHz}, \mathrm{CDCl}_{3}\right): \delta_{\mathrm{H}} 0.87\left(\mathrm{t}, 6 \mathrm{H}, \mathrm{N}\left(\mathrm{CH}_{2}\right)_{3} \mathbf{C H}_{3},{ }^{3} \boldsymbol{J}\right.$ $=7 \mathrm{~Hz}), 1.09\left(\mathrm{~s}, 12 \mathrm{H}, \mathrm{CH}_{2} \mathrm{C}(\mathbf{M e})_{2}\right), 1.32\left(\mathrm{~m}, 4 \mathrm{H}, \mathrm{N}\left(\mathrm{CH}_{2}\right)_{2} \mathbf{C H}_{2} \mathrm{CH}_{3}\right), 1.40\left(\mathrm{~m}, 4 \mathrm{H}, \mathrm{NCH}_{2} \mathbf{C H}_{2}\right)$, $2.44\left(\mathrm{~m}, 4 \mathrm{H}, \mathrm{NCH}_{2}\right), 2.48\left(\mathrm{~s}, 4 \mathrm{H}, \mathbf{C H}_{\mathbf{2}} \mathrm{C}(\mathrm{Me})_{2}\right) \cdot{ }^{13} \mathrm{C} \mathrm{NMR}\left(100 \mathrm{MHz}, \mathrm{CDCl}_{3}\right): \delta_{\mathrm{C}} 13.99$ (q, $\left.\mathrm{N}\left(\mathrm{CH}_{2}\right)_{3} \mathbf{C H}_{3}\right), 20.62$ (t, N(CH$\left.)_{2} \mathbf{C H}_{2} \mathrm{CH}_{3}\right), 27.62$ (q, $\left.\mathrm{CH}_{2} \mathrm{C}(\mathbf{M e})_{2}\right), 32.92$ (t, $\left.\mathrm{NCH}_{2} \mathbf{C H}_{2}\right), 42.02$ (t, $\mathrm{NCH}_{2}$ ), 52.84 (s, $\left.\mathrm{CH}_{2} \mathbf{C}(\mathrm{Me})_{2}\right), 52.85$ (t, $\left.\mathbf{C H}_{2} \mathrm{C}(\mathrm{Me})_{2}\right), 211.19$ (s, CO). HRMS: $\mathrm{C}_{17} \mathrm{H}_{36} \mathrm{~N}_{2} \mathrm{O}$ requires $m / z$ 285.2908. Found 285.2900.

1-Allyl-2,2,6,6-tetramethylpiperidin-4-one (3d) from 3a. To a solution of 3a (3.88 g, 25 $\mathrm{mmol})$ in hexane $(50 \mathrm{~mL})$ was added potassium carbonate $(7.0 \mathrm{~g}, 51 \mathrm{mmol})$ and allyl bromide (4.29 $\mathrm{mL}, 6.0 \mathrm{~g}, 50 \mathrm{mmol})$. The mixture was stirred at room temperature for 7 days. Thereafter, the precipitated potassium salts were filtered off, and the solvent was removed by distillation. The residue was purified by flash chromatography (silica gel, $\mathrm{Et}_{2} \mathrm{O}$ and hexane 1:1) to give $\mathbf{3 d}$ $(0.32 \mathrm{~g}, 6 \%)$ as a yellow oil, which was identical with $\mathbf{3 d}$ prepared from $\mathbf{7 d}$.

1-Benzyl-2,2,6,6-tetramethylpiperidin-4-one (3e) from 3a. When 3a was treated with benzyl bromide, as described for the reaction of $\mathbf{3 a}$ with allyl bromide, the product $\mathbf{3 e}$ (1\% yield) was formed as colorless needles, which were identical with 3e prepared from $7 \mathbf{e}$.

2,2,6,6-Tetramethyl-1-(5-methylthiazol-2-yl)piperidin-4-one (11). To a solution of 3a (153 $\mathrm{mg}, 1.0 \mathrm{mmol})$ in anhydrous THF $(2 \mathrm{~mL})$ was added $10(1.10 \mathrm{~mL}, 1.0 \mathrm{mmol}, 9.6 \%$ in THF). After stirring for $48 \mathrm{~h}$ at room temperature, the solvent was removed at reduced pressure. The crude product was purified by flash chromatography on silica gel (ether/hexane 1:3 to 1:1, v/v) to give 11 . 
11. Yellow crystalline solid, $\mathrm{mp} 84-86^{\circ} \mathrm{C}, 0.19 \mathrm{~g}$, yield 74\%; IR $\left(v_{\max }, \mathrm{cm}^{-1}\right): 2999(\mathrm{CH}), 1708$ (CO), $1528(\mathrm{C}=\mathrm{C}) .{ }^{1} \mathrm{H}$ NMR $\left(400 \mathrm{MHz}, \mathrm{CDCl}_{3}\right): \delta_{\mathrm{H}} 1.27\left(\mathrm{~s}, 12 \mathrm{H}, \mathrm{CH}_{2} \mathrm{C}(\mathbf{M e})_{2}\right), 2.40\left(\mathrm{~d},{ }^{4} \mathrm{~J}=1\right.$ $\mathrm{Hz}, 3 \mathrm{H}, \mathrm{CH}=\mathrm{CMe}), 2.54$ (s, 4H, $\left.\mathbf{C H}_{2} \mathrm{C}(\mathrm{Me})_{2}\right), 7.19$ (q, $\left.{ }^{4} \mathrm{~J}=1 \mathrm{~Hz}, 1 \mathrm{H}, \mathbf{C H}=\mathrm{CMe}\right) .{ }^{13} \mathrm{C}$ NMR $(100$ $\mathrm{MHz}, \mathrm{CDCl}_{3}$ ): $\delta_{\mathrm{C}} 12.58$ (q, $\mathrm{CH}=\mathrm{CMe}$ ), 29.90 (q, $\mathrm{CH}_{2} \mathrm{C}(\mathbf{M e})_{2}$ ), 54.90 (t, $\mathrm{CH}_{2} \mathrm{C}(\mathrm{Me})_{2}$ ), 58.70 (s, $\mathrm{CH}_{2} \mathbf{C}(\mathrm{Me})_{2}$ ), 132.70 (s, $\mathrm{CH}=\mathrm{CMe}$ ), 136.17 (d, $\mathbf{C H}=\mathrm{CMe}$ ), 166.36 (s, N=CS) 208.98 (s, CO). Anal. Calcd for $\mathrm{C}_{13} \mathrm{H}_{20} \mathrm{~N}_{2} \mathrm{OS}(252.13) \mathrm{C}, 61.87 ; \mathrm{H}, 7.99 ; \mathrm{N} 11.10 ; \mathrm{S}, 12.71 \%$. Found C, 61.49; H, $7.82 ; \mathrm{N}, 10.87 ; \mathrm{S}, 12.70 \%$.

2,2,6,6-Tetramethyl-1-(5-methylthiazol-2-yl)piperidin (12). Compound 12 was synthesized according to the procedure described for 11. Purification by flash chromatography (silica gel, ether/hexane $1: 6$ to $1: 4, \mathrm{v} / \mathrm{v}$ ) gave $\mathbf{1 2}$.

12. Yellow crystalline solid, mp 47-49 ${ }^{\circ} \mathrm{C}, 0.19 \mathrm{~g}$, yield 81\%; IR $\left(v_{\max }, \mathrm{cm}^{-1}\right): 3005,2974,2928$ $(\mathrm{CH}), 1532(\mathrm{C}=\mathrm{C}) .{ }^{1} \mathrm{H} \mathrm{NMR}(400 \mathrm{MHz}, \mathrm{CDCl} 3): \delta_{\mathrm{H}} 1.13$ (s, 12H, $\left.\mathrm{CH}_{2} \mathrm{C}(\mathbf{M e})_{2}\right), 1.54-1.57$ (m, $\left.4 \mathrm{H}, \mathbf{C H}_{2} \mathrm{C}(\mathrm{Me})_{2}\right), 1.67-1.75\left(\mathrm{~m}, 2 \mathrm{H}, \mathrm{CH}_{2} \mathrm{CH}_{2} \mathrm{CH}_{2}\right), 2.39$ (d, $\left.{ }^{4} \mathrm{~J}=1 \mathrm{~Hz}, 3 \mathrm{H}, \mathrm{CH}=\mathrm{CMe}\right), 7.18$ (q, $\left.{ }^{4} J=1 \mathrm{~Hz}, 1 \mathrm{H}, \mathbf{C H}=\mathrm{CMe}\right) .{ }^{13} \mathrm{C} \mathrm{NMR}\left(100 \mathrm{MHz}, \mathrm{CDCl}_{3}\right): \delta_{\mathrm{C}} 12.67(\mathrm{q}, \mathrm{CH}=\mathrm{CMe}), 17.72(\mathrm{t}$, $\mathrm{CH}_{2} \mathrm{CH}_{2} \mathrm{CH}_{2}$ ), 28.73 (q, $\left.\mathrm{CH}_{2} \mathrm{C}(\mathbf{M e})_{2}\right), 40.56$ (t, $\left.\mathbf{C H}_{2} \mathrm{C}(\mathrm{Me})_{2}\right), 55.10$ (s, $\left.\mathrm{CH}_{2} \mathbf{C}(\mathrm{Me})_{2}\right), 132.74$ (s, $\mathrm{CH}=\mathrm{CMe}$ ), 135.89 (d, $\mathbf{C H}=\mathrm{CMe}), 168.21$ (s, $\mathrm{N}=\mathrm{CS}$ ). Anal. Calcd for $\mathrm{C}_{13} \mathrm{H}_{22} \mathrm{~N}_{2} \mathrm{~S}$ (238.15) C, 65.50; H, 9.30; N, 11.75; S, 13.45\%. Found C, 65.09; H, 9.07; N, 11.54; S, $13.37 \%$.

\section{Acknowledgements}

We thank Dr. Manfred Neumann and Dr. Daniel Dembkowski, Evonik Industries AG, for valuable hints and supporting discussions.

\section{References}

1. (a) Guziec, Jr., F. S.; Torres, F. F. J. Org. Chem. 1993, 58, 1604. (b) Corey, E. J.; Gross, A. W. J. Org. Chem. 1985, 50, 5391.

2. (a) Keana, J. F. W. Chem. Rev. 1978, 78, 37. (b) Brede, O.; Beckert, D.; Windolph, C.; Göttinger, H. A. J. Phys. Chem. A 1998, 102, 1457.

3. (a) Hook, R. J. Ind. Eng. Chem. Res. 1997, 36, 1779. (b) Chakraborty, A. K.; Astarita, G.; Bischoff, K. B. Chem. Eng. Sci. 1986, 41, 997.

4. (a) Kurumada, T.; Ohsawa, H.; Oda, O.; Fujita, T.; Toda, T.; Yoshioka, T. J. Polym. Sci., Polym. Chem. 1985, 23, 1477. (b) Karrer, F. E. Makromol. Chem. 1980, 181, 595. (c) Dagonneau, M.; Ivanov, V. B.; Rozantsev, E. G.; Sholle, V. D.; Kagan, E. S. J. Macromol. Sci., Polym. Rev. 1982, 22, 169.

5. Dagonneau, M.; Kagan, E. S.; Mikhailov, V. I.; Rozantsev, E. G.; Sholle V. D. Synthesis 1984, 895. 
6. (a) Heintz, W. Justus Liebigs Ann. Chem. 1874, 174, 133. (b) Disteldorf, J.; Hübel, W.; Broschinski, L.; Kriebel, G. EP 0004 104, 1979; Chem. Abstr. 1980, 92, 22387. (c) Son, Y.C.; Suib, S. L.; Malz, Jr., R. E. Chemical Industries 2003, 89, 559. (d) Wu, A.; Yang, W.; Pan, X. Synth. Commun. 1996, 26, 3565.

7. (a) Guareschi, I. Atti d. R. Acc. delle Scienze di Torino 1894, 29, 680. (b) Guareschi, I. Ber. Dtsch. Chem. Ges. 1895, 28, 160. (c) Sosnovsky, G.; Lukszo, J.; Brasch, R. C.; Eriksson, U. G.; Tozer, T. N. Eur. J. Med. Chem. 1989, 24, 241.

8. Haruna, T.; Nishimura, A.; Sugibuchi, K. Eur. Pat. Appl. 0152 935, 1985; Chem. Abstr. 1986, 104, 88440.

9. Bacallao, N. L.; Cuéllar, A. C.; Konkobwa, A. M.; Dombi, M. N. Rev. Cubana Farm. 1991, $25,20$.

10. (a) Mailey, E. A.; Day, A. R. J. Org. Chem. 1957, 22, 1061. (b) Orthner, L. Justus Liebigs Ann. Chem. 1927, 456, 225.

11. Novelli, F.; Sparatore, F. Il Farmaco 2002, 57, 871.

12. (a) Lutz, W. B.; Lazarus, S.; Meltzer R. I. J. Org. Chem. 1962, 27, 1695. (b) Robertson, J. E.; Biel, J. H.; DiPierro, F. J. Med. Chem. 1963, 6, 381.

13. Heintz, W. Justus Liebigs Ann. Chem. 1880, 201, 90.

14. Simon, I. B.; Vvedenskii, V. P. J. Gen. Chem. USSR (Engl. Transl.) 1964, 34, 4096.

15. Hall, Jr., H. K. J. Am. Chem. Soc. 1957, 79, 5444.

16. Weerawarna, S. A.; Jewell, R. A. EP 1457 491, 2004; Chem. Abstr. 2004, 141, 260733.

17. Gan, H.; Whitten, D. G. J. Am. Chem. Soc. 1993, 115, 8031.

18. Anderson, C. D.; Shea, K. J.; Rychnovsky, S. D. Org. Lett. 2005, 7, 4879.

19. (a) Slongo, M.; Karrer, F. EP 0097 616, 1983; Chem. Abstr. 1984, 100, 122831. (b) Toray Industries, Inc. Jpn.; Sanyo Chemical Industries Ltd. JP 56147 769, 1981; Chem. Abstr. 1982, 96, 142712.

20. Tanikella, M. S.; Von Susani, O. U.S. Patent 3974 127, 1976; Chem. Abstr. 1977, 86, 156962.

21. (a) Banert, K.; Hückstädt, H.; Vrobel, K. Angew. Chem. 1992, 104, 72; Angew. Chem. Int. Ed. Engl. 1992, 31, 90. (b) Banert, K. Liebigs Ann./Recueil 1997, 2005. (c) Banert, K.; Targets in Heterocyclic Systems 2000, 3, 1. (d) Banert, K.; Groth, S.; Hückstädt, H.; Lehmann, J.; Schlott, J.; Vrobel, K. Synthesis 2002, 1423. (e) Banert, K.; Jawabrah AlHourani, B.; Groth, S.; Vrobel, K. Synthesis 2005, 2920. (f) Jawabrah Al-Hourani, B.; Banert, K.; Gomaa, N.; Vrobel, K. Tetrahedron 2008, 64, 5590. (g) Jawabrah Al-Hourani, B.; Banert, K.; Rüffer, T.; Walfort, B.; Lang, H. Heterocycles 2008, 75, 2667.

22. Organikum, 21st Edn.; Wiley-VCH: Weinheim, 2001; p 698. 